\title{
Efeitos da ocratoxina no desempenho do camarão-branco-do-pacífico (Litopenaeus vannamei, Bonne)
}

\author{
[The effects of ochratoxina in performance pacific white shrimp \\ (Litopenaeus vannamei Bonne)] \\ W.F. Albuquerque ${ }^{1}$, R.M. Calvet ${ }^{2}$, C.A.R. Rocha ${ }^{3}$, P.C.C. Martins ${ }^{4}$, M.M.G.P. Nóbrega ${ }^{4}$, \\ A.P. R. Costa $^{5}$, M.C.S. Muratori ${ }^{5}$ \\ ${ }^{1}$ Programa de pós-graduação - Universidade Federal do Piauí - Teresina, PI \\ ${ }^{2}$ Instituto Federal de Educação do Maranhão - Caxias, MA \\ ${ }^{3}$ Universidade Federal Rural do Rio de Janeiro - Seropédica, RJ \\ ${ }^{4}$ Universidade Federal do Rio Grande do Norte - Caicó, RN \\ ${ }^{5}$ Universidade Federal do Piauí - Teresina, PI
}

\begin{abstract}
RESUMO
A ocratoxina é um dos maiores grupos de micotoxinas; são metabólitos secundários produzidos principalmente por fungos dos gêneros Aspergillus e Penicillium. Possui propriedades tóxicas e nefrotóxicas, está relacionada à nefropatia endêmica dos Bálcãs, a tumores do trato urinário e foi classificada pela Agência Internacional de Pesquisa do Câncer (IARC) como pertencente ao grupo 2B, por ser possivelmente carcinogênica para humanos. O objetivo do presente estudo foi avaliar os efeitos da ocratoxina A (OTA) no desempenho do camarão-branco-do-pacífico (Litopenaues vannamei). O experimento foi feito simulando o manejo produtivo de uma fazenda de camarão marinho do litoral localizada em Luís Correia, Piauí. Foram utilizados cinco tratamentos com diferentes níveis de

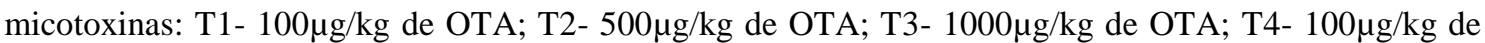
OTA e $500 \mu \mathrm{g} / \mathrm{kg}$ afatoxina $\mathrm{B}_{1}$ e T5 - $0,0 \mu \mathrm{g} / \mathrm{kg}$ de OTA. A produção de OTA foi realizada por meio da fermentação do milho, utilizando-se a cepa de Aspergillus ochraceus. Rações comerciais foram contaminadas com os núcleos de milho. A detecção e a quantificação de OTA dos núcleos, das rações comerciais e dos tecidos do camarão (cefalotórax e abdome) foram realizadas por cromatografia líquida de alta eficiência (CLAE). Para simular o sistema de criação da fazenda, os animais foram cultivados por um período de oito semanas, sendo 20 animais por caixa, recebendo alimentação duas vezes por dia. $\mathrm{O}$ menor ganho de peso observado foi no T2 e no T4 e os maiores ganhos de peso foram obtidos no T1 e no T5, que também apresentaram a melhor conversão alimentar. Após 56 dias de experimento, foi detectada OTA residual nas amostras de abdome apenas nos camarões do T1. Logo, camarões alimentados com rações contaminadas com OTA têm seu desempenho produtivo comprometido, o que gera impactos econômicos negativos para a indústria carcinicultora, além de ser um risco à saúde do consumidor, devido aos resíduos em sua musculatura.
\end{abstract}

Palavras-chave: camarão, micotoxina, desempenho produtivo, carcinicultura

\begin{abstract}
Ochratoxin A is the second largest group of mycotoxins. It is a secondary metabolite produced mainly by fungi of the genera Aspergillus and Penicillium, and has toxic and nephrotoxic properties that are associated with the Balkan endemic nephropathy and urinary tract tumors. The International Agency for Research on Cancer (IARC) classifies it as group 2B: possibly carcinogenic to humans. The aim of this study was to evaluate the effects of ochratoxin A (OTA) on the performance of the Pacific white shrimp (Litopenaues vannamei). The experiment simulated the productive management of a shrimp farm located on the marine coast of Luis Correia, Piaui State. Five treatments with different levels of mycotoxins were
\end{abstract}

Recebido em 19 de junho 2015

Aceito em 18 de fevereiro de 2016

E-mail: waleska@ufpi.edu.br 


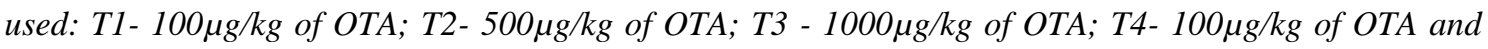
$500 \mu \mathrm{g} / \mathrm{kg}$ afatoxina $B_{1}$ and $T 5-0.0 \mu \mathrm{g} / \mathrm{kg}$ of OTA. OTA was produced by fermenting corn, using the Aspergillus ochraceus strain. Commercial feeds were contaminated with the corn kernels. OTA in the kernels, commercial feeds and shrimp tissues (cephalothorax and abdomen) were detected and quantified via high performance liquid chromatography (HPLC). To simulate the farming system, totaling 20 animals per box. The animals were fed twice a day and raised under these conditions for eight weeks. The shrimp gained weight during the weeks of the test, when subjected to different OTA treatments. The lowest weight gain was observed in T2 and T4 and the highest weight gains were in T1 and T5, which also presented the best feed conversion ratio. After 56 days, residual OTA was detected in samples of shrimp abdomen only in T1. Therefore, the productive performance of shrimp that are fed with OTAcontaminated feed is compromised, which has a negative economic impact on the shrimp industry, and is a health risk to consumers due to residues in the muscles.

Keywords: shrimp, mycotoxins, productive performance, shrimp farming

\section{INTRODUÇÃO}

As micotoxinas são metabólitos secundários tóxicos, produzidos por fungos filamentosos que frequentemente contaminam commodities usados como alimentos para animais (Hussein e Brasel, 2001; Sudakin, 2003). Mais de 80 espécies de fungos produzem mais de 300 diferentes tipos de micotoxinas em produtos alimentícios, como: arroz, milho, feijão, trigo, cevada, soja, castanha do Brasil, farelo de peixe, frutas, presunto, queijo, leite e até vinho (Cast, 2003; Zain, 2011). Entre as micotoxinas conhecidas, as aflatoxinas, as ocratoxinas, os tricotecenos, as zearalenonas, as fumonisinas, as toxinas tremorgênicas e os alcaloides de ergot têm sido investigados e recebem atenção especial por serem considerados de maior importância à saúde humana e à saúde animal (Bennett e Klich, 2003; Zain, 2011).

A ocratoxina (OTA) é um dos maiores grupos de micotoxinas, produzida principalmente por fungos dos gêneros Aspergillus: A. ochraceus, A. carbonarius, A. niger, A. westerdijkia, A. melleus, A. sclerotiorum, A. sulphureus $e$ Penicillium: $P$. verrucosum, $P$. nordicum e outras espécies (Cast, 2003; Frisvad et al., 2004; Abrunhosa et al., 2006; Tjamos et al., 2006).

A OTA (7-[L- $\beta$ fenilalanilcarbonil] carboxil-5cloro-8 hidroxi-3,4 di-hidro-3R metil isocumarina) é uma isocumarina ligada a uma molécula de fenilalanina (Remiro, 2010), que possui propriedades tóxicas e nefrotóxicas conferidas pelo caráter isocumarínico da molécula, e ao grupo carbonila cetona (Xiao et al.,1996). A ocratoxina A também está relacionada à nefropatia endêmica dos Bálcãs e aos tumores do trato urinário (Cerain et al., 2003; Pfohl-Leszkowicz et al., 2002; Ringot et al., 2006; Zain, 2011). Foi classificada pela Agência Internacional de Pesquisa do Câncer (IARC) como pertencente ao grupo $2 \mathrm{~B}$, por ser possivelmente carcinogênica para humanos (IARC, 1993).

A contaminação de alimentos e rações por OTA representa um problema de importância econômica e um grave perigo tanto para a saúde humana quanto para a saúde animal devido ao potencial de transmissão das toxinas para carne, leite e derivados (D'mello e Macdonald, 1997; Rosa et al., 2008, 2009).

Desse modo, níveis de ocratoxina presente na ração de camarões têm recebido destaque como um fator prejudicial na produção e na sanidade desses animais (Santos et al., 2010), podendo causar redução no crescimento, distúrbios fisiológicos e histológicos e alterações que resultam na redução da produtividade (Anh Tuan et al., 2002; Manning et al., 2003; Supamattaya et al., 2005). No entanto, estudos sobre a ação da OTA na saúde dos camarões são relativamente pouco considerados.

O presente trabalho tem como objetivo avaliar os efeitos da ocratoxina A no desempenho e no crescimento no camarão-branco-do-pacífico (Litopenaeusvannamei) em condições de cultivo.

\section{MATERIAL E MÉTODOS}

Este experimento foi apreciado e aprovado pelo Comitê de Ética e Experimentação com Animais da Universidade Federal do Piauí, sob o protocolo $\mathrm{N}^{\circ} 002 / 12$. O projeto foi realizado em 
uma fazenda que cultiva camarão marinho, localizada em Luís Correia, PI (2 56’09,78' S $41^{\circ} 26^{\prime} 35,41^{\prime}$ ' O), com clima tropical úmido tipo $\mathrm{AW}$, a qual cedeu o espaço físico para as instalações e o desenvolvimento experimental. Foram utilizadas as pós-larvas de Litopenaeus vannamei produzidas na fazenda em estudo.

O delineamento experimental foi inteiramente ao acaso,com cinco tratamentos, representados pelas concentrações de micotoxinas, e quatro repetições, sendo a unidade experimental caracterizada por quatro tanques contendo 20 animais por tanque.

Os tratamentos foram classificados em: T1$100 \mu \mathrm{g} / \mathrm{kg}$ de ocratoxina A (OTA); T2- $500 \mu \mathrm{g} / \mathrm{kg}$ de OTA; T3- $1000 \mu \mathrm{g} / \mathrm{kg}$ de OTA; T4- $100 \mu \mathrm{g} / \mathrm{kg}$ de OTA e $500 \mu \mathrm{g} / \mathrm{kg}$ de $\mathrm{AFB}_{1}$ e T5 - $0,0 \mu \mathrm{g} / \mathrm{kg}$ de OTA.

Para a produção de ocratoxina A, foi utilizada uma cepa de Aspergillus ochraceus adquirida na Micoteca da Empresa de Pesquisa Agropecuária de Minas Gerais (Epamig), Lavras, MG. A cepa para produção dos núcleos foi inoculada em ágar extrato de malte (MEA) e incubada em estufa a $25^{\circ} \mathrm{C}$, por aproximadamente sete dias, no laboratório de Controle Microbiológico de Alimentos do Núcleo de Estudos, Pesquisa e Processamento de Alimentos (Nueppa) da Universidade Federal do Piauí. Seguiu-se a metodología recomendada por Magnoli et al. (2011) com adaptações.

As ocratoxinas foram produzidas pela fermentação do milho utilizando-se uma cepa de A.ochraceus. O milho usado como substrato foi esterilizado e colocado em frascos tipo Erlenmeyer. Foram acrescentados em cada frasco $10 \mathrm{~mL}$ de água esterilizada, e parte da cepa do fungo foi inoculada. As culturas foram incubadas a $25^{\circ} \mathrm{C}$ durante 21 dias. No $21^{\circ}$ dia, os frascos foram autoclavados, e o material foi seco durante 48 horas a $40^{\circ} \mathrm{C}$, em um forno de ar forçado e, depois, moído a um pó fino. A extração de OTA do pó de milho foi feita usando-se a metodologia recomendada por Santos e Rodrigues-Amaya (1989). Utilizaram-se 50g do milho moído, que foi extraído com $270 \mathrm{~mL}$ de metanol PA e $30 \mathrm{~mL}$ de $\mathrm{KCl} 4 \%$. Em seguida, a solução foi agitada durante cinco minutos, em liquidificador doméstico. A mistura foi filtrada com papel-filtro Whatman no04 (Whatman, Inc., Clifton, New
Jersey, EUA), e uma alíquota de $150 \mathrm{~mL}$ foi recolhida. Em um becker, acrescentaram-se ao filtrado $50 \mathrm{~g}$ de celite (terra de infusórios) mais $150 \mathrm{~mL}$ da solução de sulfato de amônio $30 \%$ (solução clarificante). A mistura foi agitada por mais cinco minutos, em liquidificador, e o extrato foi filtrado em papel de filtro. Foram recolhidos $150 \mathrm{~mL}$ do filtrado e colocados em funil de separação junto com $150 \mathrm{~mL}$ de água destilada. Realizaram-se duas partições com $10 \mathrm{~mL}$ de clorofórmio cada. Cada partição foi agitada durante cinco minutos e deixada em repouso por cerca de três minutos, de modo que ocorresse separação das fases. A fase clorofórmica foi recolhida de ambas as partições, e um volume final de aproximadamente $10 \mathrm{~mL}$ foi recolhido. A fase clorofórmica foi evaporada em rotaevaporador, e o extrato seco foi ressuspenso em $1 \mathrm{~mL}$ de metanol e agitado em vórtex, sendo transferido para um frasco âmbar, este foi evaporado em capela de fluxo laminar.

A detecção e a quantificação da ocratoxina A foram realizadas por cromatografia líquida de alta eficiência (CLAE), utilizando-se um cromatógrafo SHIMADZU ${ }^{\circledR}$, modelo PROMINENCE, com detector de fluorescência modelo RF-10AXL SUPER, loop de $20 \mu \mathrm{L}$, de acordo com a metodologia proposta por Scudamore e McDonald (1998).

As separações cromatográficas foram realizadas em uma coluna de fase reversa (de sílica gel, 150 $\mathrm{x}$ 4,6mm id., 5,0 $\mu \mathrm{m}$ de tamanho de partículas, VARIAN, Inc. Palo Alto, EUA). A fase móvel utilizada foi acetonitrila:água:ác. acético (57:41:2 v/v/v) a uma vazão de $1,0 \mathrm{~mL} / \mathrm{min}$. A fluorescência de derivados de ocratoxina foi gravada em comprimentos de onda de excitação e emissão de $\lambda 300 \mathrm{~nm}$ e $460 \mathrm{~nm} \lambda$, respectivamente. A curva padrão foi construída em diferentes concentrações de OTA. Essa toxina foi quantificada pela correlação das alturas dos picos do extrato da amostra com o da curva padrão. O limite de detecção do método analítico foi de $0,02 \mathrm{ng} / \mathrm{g}$.

Para o preparo dos tratamentos, as rações comerciais da marca Fri-Ribe para camarão foram analisadas para detecção de OTA. As amostras que apresentaram $0,0 \mu \mathrm{g} / \mathrm{kg}$ de OTA foram utilizadas como controle negativo $(\mathrm{CN})$. Aquelas que apresentaram contaminação foram selecionadas para os ajustes das dosagens para 
formulação dos tratamentos. A metodologia usada para extração da micotoxina foi a descrita por Santos e Rodrigues-Amaya (1989), conforme relatado anteriormente.

A detecção e a quantificação de OTA foram realizadas por CLAE, seguindo a metodologia proposta por Scudamore e Mcdonald (1998).

As rações que apresentaram algum nível de contaminação foram acrescidas do núcleo previamente obtido, e as dosagens ajustadas nas concentrações de 100, 500 e 1000ppb de ocratoxina A para os tratamentos T1, T2, T3. O T4 (CP) foram elaboradas e constituídas de $100 \mu \mathrm{g} / \mathrm{kg}$ de OTA e $500 \mu \mathrm{g} / \mathrm{kg}$ de $\mathrm{AFB}_{1}$.

A ração selecionada foi moída, fracionada em quatro grupos de $6,0 \mathrm{~kg}$ e acrescida de núcleos de milho contendo OTA conforme a dose do tratamento. Posteriormente, esses grupos foram homogeneizados em baldes com sistemas de vedação para evitar contaminação do manipulador.

Após a adição dos núcleos, a ração foi analisada para confirmar a quantidade de OTA presente, por meio da CLAE, verificando-se, assim, se a quantidade de toxina desejada foi alcançada. Logo após confirmação da quantidade de toxina contida nas rações, estas foram armazenadas em temperatura ambiente até o início dos testes in vivo.

Juvenis de Litopenaeus vannamei, com aproximadamente $2,5 \mathrm{~g}$ de peso, foram distribuídos aleatoriamente em 20 tanques (caixas d'águas de polietileno com capacidade para 100 litros). Os camarões foram mantidos em um sistema de fluxo contínuo de 2,0 $\mathrm{L} \mathrm{min}^{-1}$ de vazão de água. Cada tanque tinha densidade de 25,6 animais $/ \mathrm{m}^{2}$, totalizando 20 animais por tanque.

Cada tanque possuía três bandejas para alimentação com diâmetro de $50 \mathrm{~mm}$, onde era colocada a ração para os animais. $\mathrm{O}$ sistema de aeração foi acoplado ao soprador central do berçário da fazenda, ligado em cada tanque por mangueiras 24 horas por dia. Foi realizada semanalmente a biometria dos camarões e foram observados os aspectos morfológicos dos animais de cada tratamento.
Os camarões foram adaptados às condições experimentais por 10 dias; durante esse período, receberam alimentação com $0,0 \mu \mathrm{g} / \mathrm{kg}$ de OTA. Após, iniciou-se o experimento em que os camarões eram alimentados duas vezes por dia, conforme o manejo produtivo da fazenda. A ração em pó contaminada com OTA era homogeneizada e transformada em bolos com aproximadamente $20 \mathrm{~g}$ cada. Esses bolos eram colocados nas bandejas duas vezes por dia, simulando o manejo da propriedade.

Antes do novo arraçoamento, os restos de alimentação presentes nas bandejas eram despejados em baldes contendo solução de cloro a 5,0ppm, para neutralizar a OTA residual presente. A cada dois dias, realizava-se a sifonagem para limpeza dos resíduos presentes no fundo da caixa. Os animais foram criados nessas condições durante oito semanas, simulando o ciclo de produção comercial das fazendas carcinicultoras do Piauí. O peso dos animais do estudo in vivo (controle negativo) foi comparado ao peso dos animais do viveiro, que recebeu as mesmas pós-larvas deste experimento.

Com o término dos experimentos, os animais restantes foram mortos mediante insensibilização no gelo, acondicionados em sacos plásticos estéreis de acordo com os tratamentos, acondicionados em caixas isotérmicas com gelo e encaminhados ao laboratório de Controle Microbiológico de Alimentos do Nueppa para análise residual de OTA no cefalotórax e no abdome, os quais foram separados de forma asséptica. Realizou-se um pool amostral de cada região (cefalotórax e abdome) dos animais de acordo com cada tratamento.

A extração e a quantificação de OTA no cefalotórax e no abdome dos animais submetidos aos diferentes tratamentos foram realizadas segundo metodología descrita por Santos e Rodrigues-Amaya (1989) e Scudamore e Mcdonald (1998), respectivamente.

Todos os dados foram submetidos à análise de variância e ao teste de SNK para se determinarem as diferenças de médias $(\mathrm{P}<0,05)$. Os efeitos tóxicos da ocratoxina A sobre o crescimento e o desempenho também foram estimados pela análise de regressão linear. Todas as análises estatísticas foram realizadas 
utilizando-se o programa Statistical Analysis Sistem (SAS, 2000).

\section{RESULTADOS}

O peso médio aumentou linearmente dentro do mesmo tratamento e em todos os tratamentos com o passar das semanas, e as maiores médias de ganho de peso foram obtidas nos tratamentos 1 e $5(\mathrm{CN})$, aos quais eram administradas doses de $100 \mu \mathrm{g} / \mathrm{kg}$ e $0,0 \mu \mathrm{g} / \mathrm{kg}$ de OTA (Tab. 1), no entanto não houve diferença significativa $(\mathrm{P}>0,05)$ entre os tratamentos. Pode-se observar que houve diferença $(\mathrm{P}<0,05)$ entre as semanas avaliadas quanto ao ganho de peso dos camarões submetidos a diferentes tratamentos de OTA (Tab. 1).

O T5 bem como o T1 tiveram a melhor conversão alimentar e o menor tempo de cultivo (Tab. 2).

Tabela 1. Média de ganho de peso dos camarões por semana submetidos a diferentes concentrações de ocratoxina A durante oito semanas

\begin{tabular}{cccccc|c}
\hline \multicolumn{7}{c}{${\text { Média de ganho de peso }(\mathrm{g})^{\mathrm{a}}}^{\mathrm{a}}$ Tratamentos $(\mu \mathrm{g} / \mathrm{kg}$ OTA $)$} \\
\hline Semanas & T1 & T2 & T3 & T4 & T5 & Média \\
\hline 1 & 1,9 & 2,0 & 2,1 & 1,7 & 2,1 & $1,9^{\mathrm{a}}$ \\
2 & 1,5 & 0,9 & 0,0 & 1,5 & 0,4 & $0,9^{\mathrm{b}, \mathrm{c}}$ \\
3 & 0,7 & 1,1 & 2,2 & 0,4 & 1,7 & $1,2^{\mathrm{b}}$ \\
4 & 0,2 & 0,5 & 0,2 & 0,4 & 0,4 & $0,3^{\mathrm{c}}$ \\
5 & 0,9 & 0,2 & 0,1 & 0,5 & 0,0 & $0,3^{\mathrm{c}}$ \\
6 & 0,6 & 0,5 & 0,9 & 0,5 & 1,1 & $0,7^{\mathrm{b}, \mathrm{c}}$ \\
7 & 0,5 & 0,4 & 0,1 & 0,4 & 0,7 & $0,4^{\mathrm{c}}$ \\
8 & 1,0 & 1,2 & 0,4 & 1,1 & 0,9 & $0,9^{\mathrm{b}, \mathrm{c}}$ \\
\hline Média & $0,9^{\mathrm{a}}$ & $0,8^{\mathrm{a}}$ & $0,7^{\mathrm{a}}$ & $0,8^{\mathrm{a}}$ & $0,9^{\mathrm{a}}$ & \\
\hline
\end{tabular}

Coeficiente de variação (\%) 95,9

${ }_{\mathrm{a}}^{\mathrm{g}}=$ grama; ${ }^{\mathrm{b}} \mathrm{T} 1=100 \mu \mathrm{g} / \mathrm{kg}$ OTA; ${ }^{\mathrm{c}} \mathrm{T} 2=500 \mu \mathrm{g} / \mathrm{kg}$ OTA; ${ }^{\mathrm{d}} \mathrm{T} 3=1000 \mu \mathrm{g} / \mathrm{kg}$ OTA.

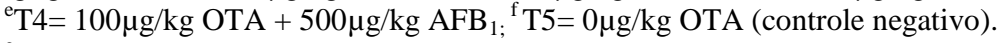

${ }^{\mathrm{g}}$ Equação linear: médias seguidas de letras diferentes, na mesma coluna, diferem estatisticamente $(\mathrm{P}<0,05 \%)$.

${ }^{\mathrm{h}}$ Médias seguidas da mesma letra, na mesma linha, não diferem estatisticamente ( $\left.\mathrm{P}>0,05 \%\right)$.

Tabela 2. Conversão alimentar de camarões alimentados com rações contendo diferentes concentrações de ocratoxina A

\begin{tabular}{|c|c|c|c|c|c|}
\hline Tratamento & $\begin{array}{c}\text { Concentração de } \\
\text { ocratoxina A } \\
(\mu \mathrm{g} / \mathrm{kg})\end{array}$ & $\begin{array}{c}\text { Conversão } \\
\text { alimentar } \\
\text { aparente }\end{array}$ & $\begin{array}{l}\text { Período de cultivo } \\
\text { estimado para atingir } \\
10 \mathrm{~g} * \text { (dias de cultivo) }\end{array}$ & $\begin{array}{l}\text { Quantidade de } \\
\text { ração consumida } \\
(\mathrm{kg})\end{array}$ & $\begin{array}{l}\text { Custos com } \\
\text { ração (R\$) }\end{array}$ \\
\hline $\mathrm{T} 1^{\mathrm{a}}$ & 100 & 1,04 & 86 & 772,11 & $+96,25$ \\
\hline $\mathrm{T} 2^{\mathrm{b}}$ & 500 & 1,09 & 101 & 906,78 & $+460,56$ \\
\hline $\mathrm{T} 3^{\mathrm{c}}$ & 1000 & 1,23 & 105 & 942,70 & $+557,55$ \\
\hline $\mathrm{T} 4^{\mathrm{d}}$ & $100+500 \mathrm{AFLA}_{1}$ & 1,15 & 100 & 987,80 & $+679,32$ \\
\hline $\mathrm{T} 5^{\mathrm{e}}$ & 0 & 1,01 & 82 & 736,20 & $1.987,74$ \\
\hline
\end{tabular}

Foram detectados níveis de $0,20 \mu \mathrm{g} / \mathrm{kg}$ de OTA apenas na região do abdome dos camarões submetidos à dieta com doses de $100 \mathrm{ug} / \mathrm{kg}$ de OTA (T1) por um período de oito semanas (Tab.
3). Resíduos de OTA nas amostras de cefalotórax e abdome dos camarões alimentados com os demais tratamentos não foram detectados. 
Efeitos da ocratoxina...

Tabela 3. Níveis de OTA no abdome e no cefalotórax dos camarões submetidos a diferentes tratamentos com OTA durante oito semanas

\begin{tabular}{|c|c|c|c|c|}
\hline Amostra & $\begin{array}{l}\text { Tempo de } \\
\text { retenção }\end{array}$ & Área & Altura & $\begin{array}{l}\text { Níveis de ota } \\
\qquad(\mu \mathrm{G} / \mathrm{KG})\end{array}$ \\
\hline $\mathrm{T}^{\mathrm{a}}{ }^{\mathrm{a}} \mathrm{OT}$ abd 1 & 5,240 & 14598,7 & 1489,4 & 0,20 \\
\hline T1OTA abd 2 & 5,238 & 14740,5 & 1529,6 & 0,21 \\
\hline T1OTA cefalot 1 & $\mathrm{ND}^{\mathrm{b}}$ & ---- & --- & --- \\
\hline T1OTA cefalot 2 & ND & ---- & ---- & ---- \\
\hline $\begin{array}{l}\text { Demais amostras }{ }^{\mathrm{c}} \\
\text { (abd;cefalot.) }\end{array}$ & ND & ---- & ---- & ---- \\
\hline
\end{tabular}

\section{DISCUSSÃO}

A contaminação de alimentos por micotoxinas em todo o mundo é um problema de importância agroeconômica. O impacto econômico das micotoxinas inclui efeitos tóxicos sobre humanos e animais, com consequente aumento nos cuidados com a saúde e nos custos verterinarios (Hussein e Brasel, 2001). Tudo isso pode se agravar caso não existam normas rígidas que imponham limites de concentração de micotoxinas nas lavouras e caso sejam mantidos os baixos padrões de qualidade de armazenamento (umidade, ratos e infestação de insetos) dos alimentos (Abdel-Aziz et al., 2010).

No presente estudo, verificou-se que os camarões (L. vannamei) alimentados com dietas contaminadas com níveis de OTA de 0,0ppb, $100 \mathrm{ppb}, 500 \mathrm{ppb}, 1000 \mathrm{ppb}$ e 100ppb de OTA + $500 \mathrm{ppb}$ de $\mathrm{AFB}_{1}$, por um período de oito semanas, não apresentaram diferença significativa de ganho de peso entre os tratamentos $(\mathrm{P}>0,05)$ : suas médias variaram de 0,7 a $0,9 \mathrm{~g}$. Esses dados não corroboram os do estudo realizado por Supamattaya et al. (2005) com camarões Penaeus monodon alimentados com dietas contendo 100ppb; 200ppb e 1000ppb de OTA durante oito semanas, em que foi observada uma ligeira redução de peso apenas nos animais alimentados com a dieta de $1000 \mathrm{ppb}$, quando comparados ao grupo controle. No presente estudo, quando se avaliaram as semanas, observou-se que houve diferença $(\mathrm{P}<0,05)$ quanto ao ganho de peso (Tab. 1), com maior expressão na terceira semana. Foi observado que as quantidades de OTA testadas não impediram o crescimento dos camarões, que ocorreu de forma gradual mesmo em diferentes doses da micotoxina.
Outras toxinas, como a aflatoxina B1 $\left(\mathrm{AFB}_{1}\right)$, quando administradas em doses de 1000ppb e $2500 \mathrm{ppb}$ por oito semanas, interferiram negativamente no ganho de peso do L. vannamei (Boonyaratpalin, 2001; Calvet, 2012). Já a deoxinivalenol (DON) também afetou o desenvolvimento do camarão-preto (Penaeus monodon) quando alimentado, também por oito semanas, com dietas com doses de 1000ppb de DON, mas este apresentou índice de desempenho significativamente superior, em comparação com o controle (Supamattaya et al., 2005), o que mostra que, dependendo da espécie de camarão bem como da toxina administrada, as reações do animal podem variar de acordo com esses parâmetros.

Na Tab. 1, após o período de um ciclo de cultivo experimental de 56 dias, os camarões dos tratamentos T3 apresentaram o menor ganho de peso $(0,7 \mathrm{~g})$. Já o ganho de peso do T5 foi igual ao $\mathrm{T} 1$, com $0,9 \mathrm{~g}(\mathrm{P}<0,05)$. Pela projeção da equação da reta para o tratamento 1 (T1 y= $\left.0,6536 \mathrm{x}+1,9464\left(\mathrm{R}^{2}=0,9478\right)\right)$, seriam necessários mais quatro dias de cultivo para atingir o mesmo peso médio do T5 (Tab. 2), portanto o T1 $(100 \mu \mathrm{g} / \mathrm{kg})$ não interferiu tanto no tratamento dos animais quando comparado ao grupo controle. Já para os tratamentos T2, T3 e T4, o tempo de cultivo aumentou em 23, 19 e 18 dias, respectivamente, em relação ao T5, o que significa um aumento no consumo de ração nos viveiros, além do aumento no ciclo dos camarões, representando um prejuízo econômico para a indústria.

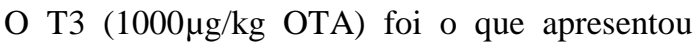
maior conversão alimentar; por conseguinte, maior tempo de cultivo, sendo este o tratamento mais prejudicial e o que apresentou maior mortalidade após o período de adaptação. 
Foi observado resíduo $(0,20 \mu \mathrm{g} / \mathrm{kg})$ de ocratoxina A apenas na musculatura dos $L$. vannamei submetidos ao tratamento com $100 \mu \mathrm{g} / \mathrm{kg}$ de OTA (T1) após as oito semanas de cultivo, nível esse que pode ser considerado perigoso para alimentação humana, pois, de acordo Commission Regulation (2006), uma dose semanal (DSA) de 120ng/kg por peso corporal é admissível; acima desses níveis, torna-se perigosa a ingesta de alimentos. Portanto, se um indivíduo de $60 \mathrm{~kg}$ consumir uma porção de $200 \mathrm{~g}$ de camarão, ele estará ingerindo, de uma só vez, cerca de $1600 \mathrm{ng} / \mathrm{kg}$ de toxina, ou seja $155 \%$ da dose diária recomendada em uma única ingesta, dado esse muito preocupante devido às propriedades imunotóxicas da OTA. Ressalta-se que a toxina foi encontrada apenas nos camarões que foram submetidos à menor dose. Isso pode ser explicado pelo fato dos animais terem a capacidade de desenvolver um mecanismo de resistência à toxina por meio da sua destoxificação ou metabolização. Assim, a toxina é convertida em uma forma menos tóxica ou inativa, sendo, em seguida, eliminada. Nesse mecanismo, podem estar envolvidas enzimas como esterases, oxidades, transferases e carboxipeptidases (Fontaínhas-Fernandes et al., 2007).

Os resultados observados neste experimento com L. vannamei foram diferentes dos obtidos por Supamattaya et al. (2005), que pesquisaram Penaeus monodon alimentados com dietas contaminadas com 100; 200 e $1000 \mu \mathrm{g} / \mathrm{kg}$ de OTA. Nesse experimento, os autores não detectaram as toxinas no cefalotórax nem na musculatura dos camarões. Essas variações podem ter ocorrido pelo fato de as pesquisas terem sido realizadas com espécies diferentes de camarões, em distintas condições de cultivo, e pela individualidade dos animais testados. Diante desses resultados e da escassez de estudos, fazem-se necessários mais estudos com ocratoxina A em L. vannamei cultivado, a fim de se verificarem possíveis alterações histopatológicas no hepatopâncreas, na glândula antenal, no intestino e em outros órgãos importantes, para fornecer subsídios aos produtores.

\section{CONCLUSÃO}

Camarões alimentados com rações contaminadas com diferentes concentrações de ocratoxina A têm seu desempenho produtivo comprometido, sendo estes extremamente sensíveis a essa toxina, o que gera impactos econômicos negativos para a indústria carcinicultora, podendo ser um risco à saúde do consumidor, devido aos resíduos encontrados em sua musculatura.

\section{AGRADECIMENTOS}

À Coordenação de Aperfeiçoamento de Pessoal de Nível Superior - Capes, pela ajuda no financiamento do projeto. À indústria Fri-Ribe S/A, pela doação das rações para o experimento. À Fazenda Secom Aquicultura, Comércio e Indústria, S/A, pelas instalações e pelos espaços físicos cedidos. Ao Núcleo de Estudos, Pesquisa e Processamento de Alimentos - Nueppa.

\section{REFERÊNCIAS}

ABDEL-AZIZ, K.B.; FARAG, I.M.; TAWFEK, N.S. et al. Saccharomyces cereviciae ameliorates oxidative stress, genotoxicity and spermatotoxic effects induced by Ochratoxin A in male Albino Mice. New York Science Journal, v. 3, p. 178190, 2010.

ABRUNHOSA, L.; SANTOS, L.; VENÂNCIO, A. Degradation of ochratoxin a by proteases and by a crude enzyme of Aspergillus niger. Food Biotechnol., v.20, p.231-242, 2006.

ANH TUAN, N.; GRIZZLE, J.M.; LOVELL, R.T. et al. Growth and hepatic lesions of Nilo tilapia (Orechromis niloticus) fed diets containing aflatoxin B1. Aquaculture, v.212, p.311-319, 2002.

BENNETT, J.W.; KLICH, M. Mycotoxins. Clin. Microbiol. Rev., v.16, p.497-516, 2003.

BOONYARATPALIN, M.; SUPAMATTAYA K.; VERAKUNPIRIYA, V.; SUPRASERT, D. Effects of aflatoxin $\mathrm{B}_{1}$ on growth performance, blood components, immune function and histopathological changes in black tiger shrimp (Penaeus monodon Fabricius). Aquacult. Res., v.32, p.388-398, 2001. 
CALVET, R.M. Efeitos da aflatoxina $B_{1}$ no desempenho do camarão branco do Pacífico (Litopenaeus vannamei). 2012. 76f. Tese (Doutorado em Ciência Animal) - Universidade Federal do Piauí, Teresina, PI.

CERAIN, L. A. Ocratoxina a: exposición en España y nuevos aspectos sobre su toxicidad. Rev. Toxicol., v.20, p.72-73, 2003.

COMMISSION Regulation (EC), 2006. N. $1881 / 2006$ of 19 December 2006. Setting maximum levels for certain contaminants in foodstuffs. Off. J. Eur. Union, Bruxelles, 364, p. 5-24. Available in: <http://eurlex.europa.eu./LexUriServ/LexUriServ.do?uri=O $\mathrm{J}: \mathrm{L}: 2006: 364: 0005: 0024: E N: P D F>$. Accessed in : 27 set. 2012.

D’MELLO, J.P.F.; MACDONALD, A.M.C. Mycotoxins. Anim. Feed Sci. Techol., v.69, p.155-166, 1997.

FONTAÍNHAS-FERNANDES, A.; PEIXOTO, F.; SANTOS, D. Biotransformation pathway in fish: an environmental review. Rev. Port. Zootec., v.1, p.11-28, 2007.

FRISVAD, J.C.; FRANK, M.; HOUBRAKEN, J.A.M.P.; KUJIPERS, A.F.A. et al. New ochratoxin A producing species of Aspergillus section Circumdati. Stud. Mycol., v.50, p.23-43, 2004.

HUSSEIN, H.S.; BRASEL, J.M. Toxicity, metabolism and impact of mycotoxins on humans and animals. Toxicology, v.167, p.101134,2001

MAGNOLI A.P.; MONGE, M.P.; MIAZZO, R.D.; CAVAGLIERI, L.R. et al. Effect of low levels of aflatoxin B1 on performance, biochemical parameters, and aflatoxin B1 in broiler liver tissues in the presence of monensin and sodium bentonite. Poult. Sci., v.90, p.48-58, 2011.

MANNING，B.B.; ULLOA，R.M.; LI，M.H.; ROBINSON, E.H. et al. Ochratoxin A fed to channel catfish (Ictalurus punctatus) causes reduced growth and lesions of hepatopancreatic tissue. Aquaculture, v.219, p.739-750, 2003.

MYCOTOXINS: risks in plant, animal and human systems. Ames: Council for Agricultural Science and Technology, 2003. n.116, 216p.
PFOHL-LESZKOWICZ, A.; PETKOVABOCHAROVA, T.; CHERNOZEMSKY, I.N.; CASTEGNARO, M. Balkan endemic nephropathy and associated urinary tract tumours: a review on aetiological causes and the potential role of mycotoxins. Food Addit. Contam., v.19, p.282, 2002

REMIRO, R.; IBÁNEZ-VEA, M.; GONZÁLEZPEÑAS, E.; LIZARRAGA, E. Validation of a liquid chromatography method for the simultaneous quantification of ochratoxin A and its analogues in red wines. J. Chromatogr., v.1217, p.8249-8256, 2010.

RINGOT, D.; CHANGO, A.; SCHNEIDER; Y. J.; LARONDELLE, Y. Toxicokinetics and toxicodynamics of ochratoxin A, an update. Chem. Biol. Interact., v.159, p.18-46, 2006.

ROSA, C.A.R.; CAVAGLIERI, L.R.; RIBEIRO, J.M.M.; KELLER, K.M. et al. Mycobiota and naturally-occurring ochratoxin A in dairy cattle feed from Rio de Janeiro state, Brazil. World Mycotoxin J., v.1, p.195-201, 2008.

ROSA, C.A.R.; KELLER, K.M.; KELLER, L.A.M.; PEREYRA, M.L.G. et al. Mycological survey and ochratoxin A natural contamination of swine feedstuffs in Rio de Janeiro state, Brazil. Toxicon, v.53, p.283-288, 2009.

SANTOS, G.A.; RODRIGUES, I.; STARKL, V.; NAEHRER, K. et al. Mycotoxins in aquaculture: occurrence in feeds components and impact on animal performance. avances em nutrición acuícola. In: SIMPOSIO INTERNACIONAL DE NUTRICIÓN ACUÍCOLA, 10., Monterrey. Proceedings... Monterrey: Universidad Autónoma de Nuevo, 2010. p.502-513.

SOARES, L.M.V.; RODRIGUEZ-AMAYA, D.B. Survey of aflatoxins, ochratoxin A, zearalenone, and sterigmatocystin in some Brazilian foods by using multi-toxin thin-layer chromatographic method. J Assoc Off Anal Chem.,v.72, p.22-6, 1989.

SAS. Statistical analysis system. Version 6.1, Cary: SAS Institute, 2000.

SCUDAMORE, K.A.; MACDONALD, S.J. A collaborative study of an HPLC method for determination of ochratoxin $\mathrm{A}$ in wheat using immunoaffinity column clean-up. Food Addit. Contam., v.15, p.401-410, 1998. 
SOME naturally occurring substances: food items and constituents, heterocyclic aromatic amines and mycotoxins. Lyon: IARC, 1993. v.56. 571p. Available in: <http://monographs.iarc.fr/ENG/Monographs/vol 56/volume56.pdf $>$. Accessed in: 24 set. 2012.

SUDAKIN, D.L. Trichothecenes in the environment: relevance to human health. Toxicol. Lett., v.143, p.97-107, 2003.

SUPAMATTAYA, K.; SUKRAKANCHANA, N.; BOONYARATPALIN, M.; SCHATZMAYR, D. et al. Effects of ochratoxin A and deoxynivalenolon growth performance and immune physiological parameters in black tiger shrimp (Penaeus monodon). J. Agric. Sci. Technol., v.27, p.91-99, 2005.
TJAMOS, S.E.; ANTONIOU, P.P.; TJAMOS, E.C. Aspergillus spp., distribution, population composition and ochratoxin A production in wine producing vineyards in Greece. Int. J. Food Microbiol., v.111, p.61-66, 2006.

XIAO, H.; MADHYASTHA, S.; MARQUARDT, R.R.; LI, S. Toxicity of ochratoxin A, its opened lactone form and several of its analogs: estructure-activity relationships. Toxicol. Appl. Pharmacol., v.137, p.182-192, 1996.

ZAIN, M.E. Impact of mycotoxins on humans and animals. J. Saudi Chem. Soc., v.15, p.129144, 2011. 